\title{
Comparative Gene Expression Analysis within Mouse Macrophage for Identifying Critical Pathways in Macrophage and Brucella suis
} Interaction

\author{
Jing Hu, ${ }^{1}$ Tonglian Wang, ${ }^{2}$ Hongbo Zhao, ${ }^{3}$ Yuzhu Song, ${ }^{2}$ Qinqin Han, ${ }^{2}$ Jinyang Zhang, ${ }^{2}$ Tao Shou, ${ }^{1}$ Fan \\ Zhang, ${ }^{4}$ Xueshan Xia, ${ }^{2}$ and Qiang Chen ${ }^{2, *}$ \\ ${ }^{1}$ Medical Oncology, The First People's Hospital of Yunnan Province, Kunming, P.R. China \\ ${ }^{2}$ Research Center of Molecular Medicine of Yunnan Province, Faculty of Life Science and Technology, Kunming University of Science and Technology, Kunming, P.R. China \\ ${ }^{3}$ Institute of Molecular and Clinical Medicine, Kunming Medical University, Kunming, P.R. China \\ ${ }^{4}$ Department of Gastroenterology, The Third People's Hospital of Yunnan Province, Kunming, P.R. China \\ "Corresponding author: Qiang Chen, Research Center of Molecular Medicine of Yunnan Province, Faculty of Life Science and Technology, Kunming University of Science and \\ Technology, No. 727 of South Jingming Road, Chenggong New Town, Kunming, 650500, P.R. China. Tel: +86-87165939528, Fax: +86-87165939528, E-mail: chq@sjtu.edu.cn
}

Received 2017 April 01; Revised 2017 August 21; Accepted 2017 August 28.

\begin{abstract}
Background: Brucella spp. are Gram-negative bacteria that cause a zoonotic disease called brucellosis in humans as well as many animals. Brucella suis (B.suis) is one of the greatest threats to the human health and food safety. Studying macrophage and B. suis interaction is critical for understanding the chronic infection mechanism. However, the interaction mechanisms, especially for molecular events triggered by B. suis infected macrophage, such as biological pathways, are still obscure.

Objectives: We will use gene set enrichment analysis (GSEA) to microarray in an attempt to find critical pathways in the interaction of macrophage and B. suis.

Methods: We applied a standardized microarray preprocessing and GSEA to 2 independent macrophage and B. suis interaction studies including smooth virulent B. suis strain 1330 (S1330) data sets and rough attenuated B. suis strain VTRS1 (VTRS1) data sets. Integrative analysis was used to find critical pathways for 2 independent macrophage and $B$. suis interaction data sets.

Results: The results demonstrated that for S1330 data sets, 8 and 13 common up- and down- regulated pathways were found in 4 interaction stages including $4 \mathrm{~h}, 8 \mathrm{~h}, 24 \mathrm{~h}$, and $48 \mathrm{~h}$ post macrophage infected S1330 B. suis, and for VTRS1 data sets, we found 30 and 19 common up- and down- regulated pathways. Comparing the results of S1330 and VTRS1 data sets, 6 and 8 common up-and downregulated pathways were identified.

Conclusions: The study of macrophage and B. suis interaction through pathway analysis highlighted genes weakly connected to the phenotype, and discovered common critical pathways in the process of macrophages and different phenotypes of B. suis interaction. The identified pathways will shed light on the understanding of the functional events within macrophage post infected $B$. suis.
\end{abstract}

Keywords: GSEA, Macrophage and B. suis Interaction, Microarray, Pathway Analysis

\section{Background}

Brucella spp., a Gram-negative and facultative intracellular bacteria, causes a zoonotic disease called brucellosis in humans and many animals including domestic pigs, cattle, wildlife camels, and so on by chronic macrophage infection (1). In 10 species reported (2), Brucella suis is one of 3 pathogenic bacteria (B. melitensis, B. suis and B. abortus) that are the biggest threat to human health and food safety. In many types of cells attacked, macrophages are the primary target cells attacked (3). Further studying the macrophage and B. suis interaction will contribute to understanding the pathogenesis.

To date, a large number of interaction studies showed that the fate of macrophages infected were modulated by Brucella. Rough Brucella organisms induce caspase2mediated macrophage death $(1,4)$, whereas smooth Brucella organisms inhibit macrophage apoptosis by inhibiting mitochondrial cell death pathway and caspase activation $(4,5)$. These results suggest that inhibition of macrophage death provide a hospitable intracellular niche for Brucella survival, while death induction promote Brucella release and dissemination (6-8). In addition, some studies observed that smooth Brucella dissociated into rough mutants that were cytotoxic to macrophage in macrophage and Brucella interaction (6), which suggested that smooth and rough Brucella should be synergistically responsible for macrophage egress and bacterial dissemination.

Despite extensive researches, the molecular events

Copyright (c) 2017, Jundishapur Journal of Microbiology. This is an open-access article distributed under the terms of the Creative Commons Attribution-NonCommercial 4.0 International License (http://creativecommons.org/licenses/by-nc/4.0/) which permits copy and redistribute the material just in noncommercial usages, provided the original work is properly cited. 
such as critical biological pathways within macrophage in macrophage and $B$. suis interaction are not yet uncovered. Performing pathway analysis to gene expression profiles data from microarray will contribute to interpreting the results to insight into biological mechanisms including bacterial invasion, survival, and replication within macrophages.

\section{Objectives}

Gene set enrichment analysis (GSEA), a gene set analysis method including pathway analysis $(9,10)$, has been widely used to identify the significant differences between pre-defined gene sets in expression from controls and cases. Here, we will use GSEA to microarray in an attempt to find critical pathways, especially common critical pathways within macrophages in the interaction of macrophages and smooth or rough B. suis.

\section{Methods}

\subsection{Datasets}

The interaction of macrophage and B. suis microarray datasets were searched and downloaded from NCBI GEO database (http://www.ncbi.nlm.nih.gov/geo/). Finally, data set GSE21117 (1), including 2 subsets of interaction of murine macrophage line and smooth or rough $B$. suis strains, was used in this study. Two phenotypes of B. suis were smooth virulent $B$. suis strain 1330 , for short $\$ 1330$ and rough attenuated $B$. suis strain VTRS1, for short VTRS1. For each subset in this study, data sets included 4 infection stages that respectively were $4 \mathrm{~h}, 8 \mathrm{~h}, 24 \mathrm{~h}$, and $48 \mathrm{~h}$ post infection, and oh post infection (uninfected group) was for control. Each phenotype or stage was treated as an independent data set. 8 case-control data sets including 4 smooth phenotype of case-control data sets and 4 rough phenotype of case-control data sets were included, and each data set was individually performed GSEA. The related information regarding the datasets such as cell line, sample size, and infection time, were listed in Table 1.

\subsection{Data Preprocessing}

Microarray data preprocessing was performed using software packages developed by Bioconductor in version 3.4 (http://www.bioconductor.org) (11) and R language in version 3.3.2 (http://www.r-project.org). All datasets were background adjusted, normalized, and log2 probe-set intensities calculated using the robust multichip averaging (RMA) algorithm in version 1.52 .0 affy package $(12,13)$. Some identifying genes failed to map any KEGG pathways that were excluded from the further analysis. The data variability was measured using the interquartile range (IQR), and a cut-off was set from the resulting distribution of IQR values for all genes. Genes with IQR values under 0.5 were excluded. When multiple probe sets targeted the same gene, the probe set with the largest variability was kept for the next analysis. Pathway analysis was separately performed for each data set.

\subsection{Gene Set Enrichment Analysis of Pathways}

Gene set enrichment analysis was performed using the version 2.40.0 Category package in the Bioconductor project and the performing GSEA's purpose was to determine whether the members of a gene set $S$ distributes randomly throughout the whole reference gene list L or was just primarily found at the top or bottom. A bigger merit of GSEA lies in the relative robustness to noise and outliers in the data. However, the fact is that a gene set including a large enough number of genes, typically 10 or more than 10 , is true. Therefore, the gene set with less than 10 genes was removed.

\subsection{Statistical Analysis}

The t-statistic mean of the genes was computed in each remaining pathway. A permutation test was implemented 1000 times, and the pathways with $P$ value $\leq 0.05$ were identified to significantly change (14).

\section{Results}

4.1. Significant Pathways Identified Within Macrophages and 2 Phenotypes of B. suis Interaction

We compared 4 data sets to identify the significant pathways within macrophage infected S1330 B. suis. The reanalysis results for these data sets were showed in Table 2. Base on the permutation 0.05 P values, we found 21, 15, 56, and 91 up-regulated pathways and 36, 81, 48, and 64 downregulated pathways in 4 interaction stages including $4 \mathrm{~h}$, $8 \mathrm{~h}, 24 \mathrm{~h}$, and $48 \mathrm{~h}$ post macrophage infected 51330 B. suis, separately. The overlap analysis showed that 8 common up-and 13 common down-regulated pathways were found. The overlaps of these pathways identified were showed in Figure $1 \mathrm{~A}$ and 1B. We performed the same analysis for VTRS1 data sets as $\$ 1330$ data sets, and the reanalysis results for these data sets also were showed in Table 2 . We found 47 , 44,68 , and 85 up-regulated pathways and $28,104,88$, and 74 down-regulated pathways, separately. The overlaps among these pathways identified showed that 30 common up-and 19 common down- regulated pathways were found. The overlaps of these pathways were showed in Figure $1 \mathrm{C}$ and 1D. 
Table 1. Experimental Design and Related Information of GSE21117 Microarray

\begin{tabular}{|c|c|c|c|c|c|}
\hline Cell Line & Brucella Strain & Chip Platform & Probes & Time Post Infection (h) & No. of Arrays \\
\hline \multirow{4}{*}{ J774.A1 cells ${ }^{\mathrm{a}}$} & \multirow{4}{*}{ B. suis strain $1330(\mathrm{~S} 1330)^{\mathrm{b}}$} & \multirow{4}{*}{ GPL1261 } & \multirow{4}{*}{$45 \mathrm{k}$} & $O(\text { Control })^{c}$ & 3 \\
\hline & & & & 4 & 3 \\
\hline & & & & 8 & 3 \\
\hline & & & & 24 & 3 \\
\hline \multirow{5}{*}{ J774.A1 cells ${ }^{\mathrm{a}}$} & \multirow{5}{*}{$\begin{array}{l}\text { B. suis strain VTRS1 } \\
{\text { (VTRS1 })^{\mathrm{b}}}^{\text {(VT) }}\end{array}$} & \multirow{5}{*}{ GPL1261 } & \multirow{5}{*}{$45 \mathrm{k}$} & 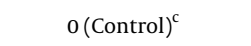 & 3 \\
\hline & & & & 4 & 3 \\
\hline & & & & 8 & 3 \\
\hline & & & & 24 & 3 \\
\hline & & & & 48 & 3 \\
\hline
\end{tabular}

a J774.A1 cells are murine macrophage-like cells.

${ }^{\mathrm{b}}$ S1330 and VTRS1 are represented the smooth virulent $B$. suis strain and rough attenuated B. suis strain, respectively.

${ }^{\mathrm{C}} \mathrm{oh}$ post infection is control that shows that macrophages are uninfected.

Table 2. Analysis Results of Differentially Expressed Pathway Number

\begin{tabular}{|c|c|c|c|c|c|}
\hline Classes & Process $(h)$ & $\begin{array}{l}\text { No. of Genes After } \\
\text { Preprocessing }\end{array}$ & $\begin{array}{l}\text { No. of Pathways Have } \\
\text { Genes } \geq 10\end{array}$ & $\begin{array}{c}\text { Up-Regulated Pathway } \\
\text { Number }\end{array}$ & $\begin{array}{l}\text { Down-Regulated } \\
\text { Pathway Number }\end{array}$ \\
\hline \multirow{4}{*}{ S1330 strain ${ }^{a}$} & $4^{\mathrm{b}}$ & 1816 & 68 & 21 & 36 \\
\hline & 8 & 2861 & 108 & 15 & 81 \\
\hline & 24 & 5552 & 160 & 56 & 48 \\
\hline & 48 & 6268 & 165 & 91 & 64 \\
\hline \multirow{4}{*}{ VTRS1 strain ${ }^{a}$} & 4 & 3578 & 107 & 47 & 28 \\
\hline & 8 & 5839 & 159 & 44 & 104 \\
\hline & 24 & 5433 & 159 & 68 & 88 \\
\hline & 48 & 5996 & 164 & 85 & 73 \\
\hline
\end{tabular}

${ }^{a}$ S1330 and VTRS1 are represented the smooth virulent $B$. suis strain and rough attenuated $B$. suis strain, respectively.

$4 \mathrm{~h}, 8 \mathrm{~h}, 24 \mathrm{~h}$ and $48 \mathrm{~h}$ are represented time post murine macrophage-like J774.A1 cells infected B. suis.

4.2. Integrative Analysis of 2 Phenotypes of B. suis and Macrophages Interaction

We compared the significant pathways of 2 phenotypes of B. suis and macrophages interaction. The significant pathways identified and overlaps were showed in Figure 2 at the same stage post infection. We found 18, 13, 14, and 59 common up-regulated and 15, 62, 40, and 45 downregulated pathways in 4 interaction stages including $4 \mathrm{~h}$, $8 \mathrm{~h}, 24 \mathrm{~h}$, and $48 \mathrm{~h}$ post infection, respectively. For 2 datasets in the 4 stages of interaction, we detected that 6 common significant pathways were up-regulated and 8 common significant pathways were down-regulated (Figure 3 ). The integrative results were listed in Table 3. In the common significant pathways, functional protein association networks of sum set of genes within the up-regulated and down-regulated pathways were showed in Figure 4.

\subsection{Dynamical Variation of Critical Significant Pathways}

From Table 3, we found some critical pathways related to immune system, immune disease and cell growth and death in common significant pathway list. These pathways included up-regulated toll-like receptor signaling pathways, RIG-I-like receptor signaling pathway, cytosolic DNAsensing pathway as well as rheumatoid arthritis pathway, and down-regulated cell cycle pathway. We analyzed the dynamical change of these pathways in total interaction stages from $4 \mathrm{~h}$ to $48 \mathrm{~h}$ post infection. The results were presented in Figure 5. From Figure 5, for S1330 and VTRS1 strains, as a whole, in early interaction stages these critical pathways within macrophage had statistical significance $(\mathrm{P}<0.05)$, most up-regulated pathways presented 
A

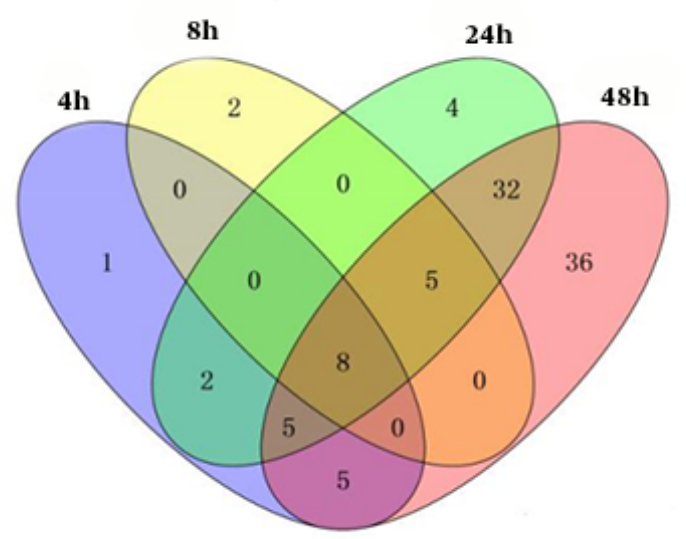

C

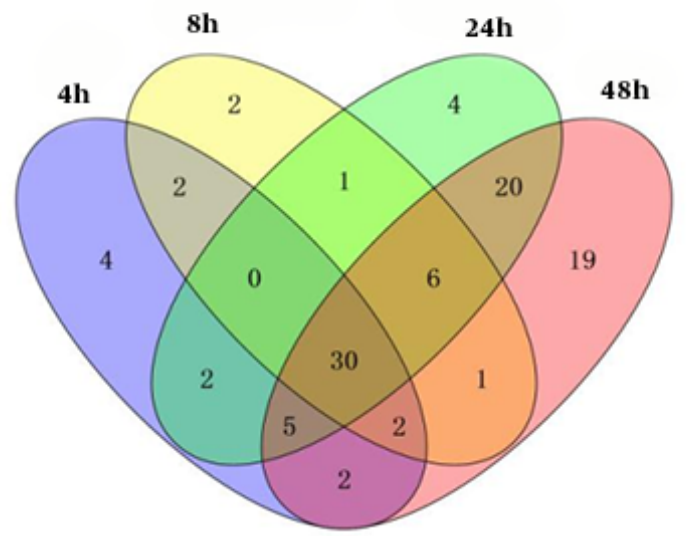

B

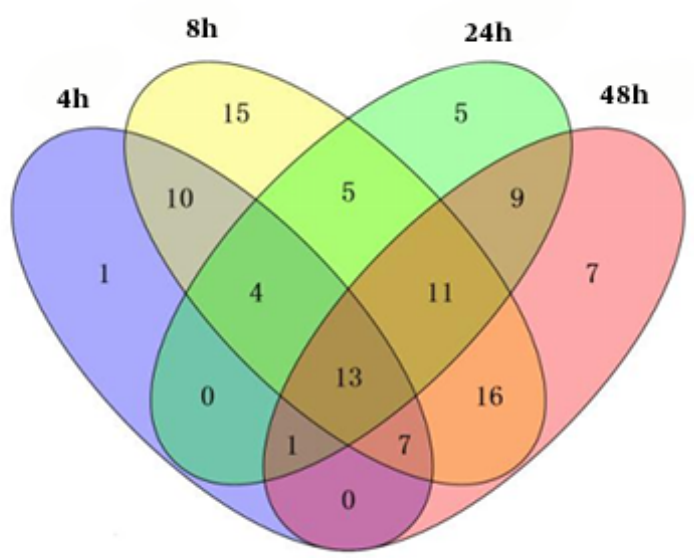

D

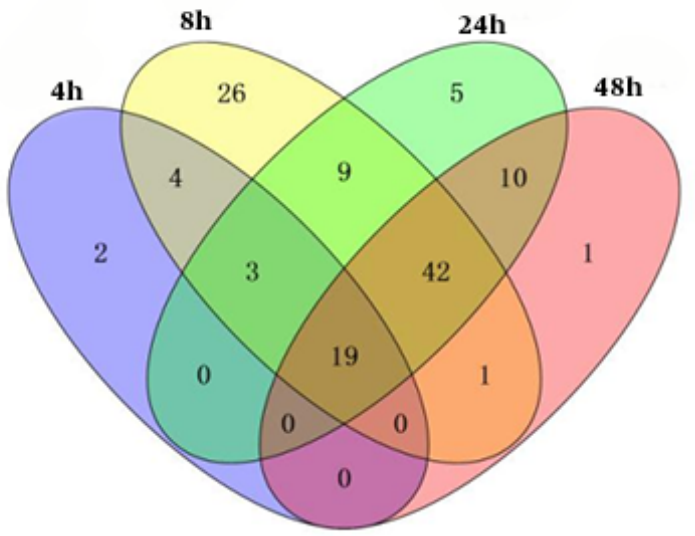

$4 \mathrm{~h}, 8 \mathrm{~h}, 24 \mathrm{~h}$ and $48 \mathrm{~h}$ are respectively represented $4 \mathrm{~h}, 8 \mathrm{~h}, 24 \mathrm{~h}$ and $48 \mathrm{~h}$ post macrophage infected $B$. suis. For each infection stage, we performed GSEA to generate p-values for each pathway and used a permutation test with 1000 times, and obtained the significant pathways with P values cut-off of $\leq 0.05$. A, GSEA detected $21,15,56$ and 91 up-regulated pathways and 8 common were found in macrophage and S1330 B. suis interaction; b, GSEA detected 36, 81, 48 and 64 down-regulated pathways and 13 common were found in macrophage and S1330 B. suis interaction; C, GSEA detected 47, 44, 68 and 85 up-regulated pathways and 30 common were found in macrophage and VTRS1 B. suis interaction; D, GSEA detected 28,104, 88 and 73 down-regulated pathways and 19 common were found in macrophage and VTRS1 B. suis interaction.

continuously to be up-regulated, than be down-regulated. Down-regulated cell cycle pathway was sequentially downregulated.

\section{Discussion}

The host macrophages are the primary targets attacked (3). Studying macrophage and $B$. suis interaction is critical for the establishment of B. suis infection models (1). In the process of macrophage and B. suis interaction, many mechanisms are responsible for the successful infection
(15). However, most mechanisms are still obscure. The microarrays are a very powerful tool to illustrate the infection mechanisms $(1,4,5,16,17)$. For the traditional single gene analysis method, the statistical analysis can only identify larger differently expressed genes, however, not find genes that made subtle contributions (10). In addition, the roles of identified genes need to be further discussed. Pathway analysis is able to put stress on genes weakly related to the phenotype and identify subtle genes by using univariate statistics (14).

In this study, we performed GSEA for 2 sets of 
A

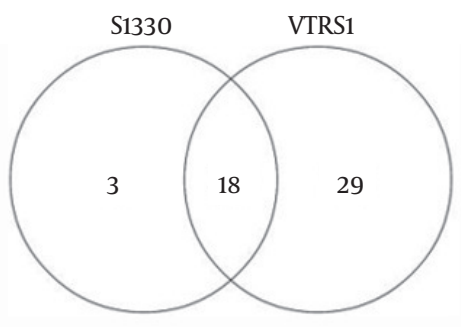

D

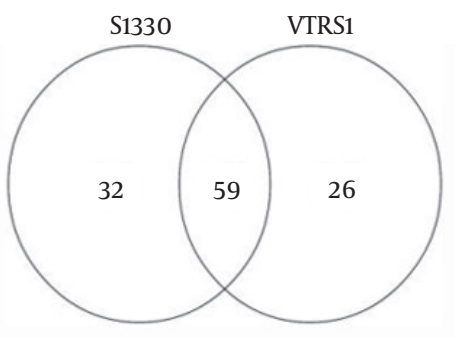

B

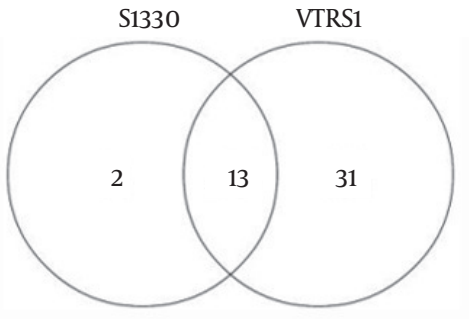

E

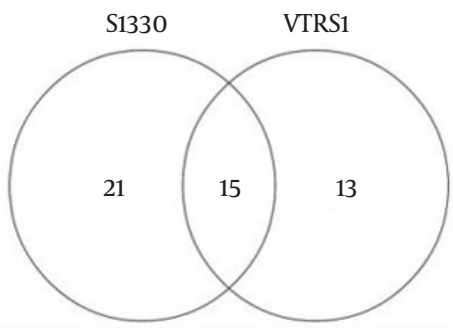

C

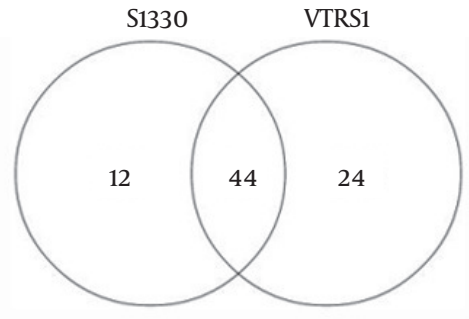

F

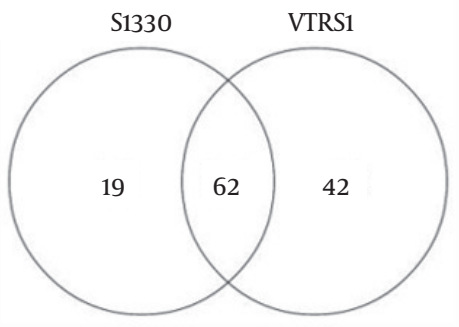

G

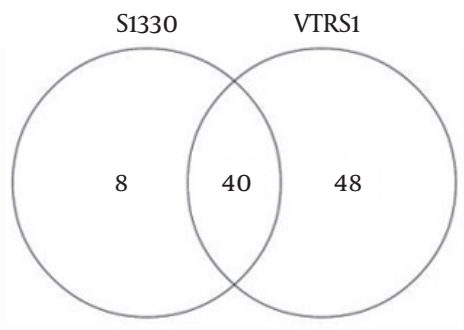

H

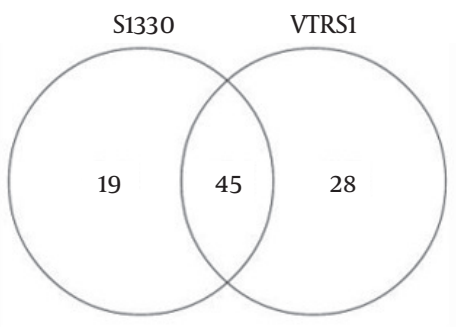

S1330 and VTRS1 are respectively represented significant pathways identified of macrophages infected between S1330 and VTRS1 B. suis. A, B, C and D, showed the significant up-regulated pathways identified and overlap at $4 \mathrm{~h}, 8 \mathrm{~h}, 24 \mathrm{~h}$ and $48 \mathrm{~h}$ post infection, respectively; $\mathrm{E}, \mathrm{F}, \mathrm{G}$ and $\mathrm{H}$, showed the significant down-regulated pathways identified and overlap at $4 \mathrm{~h}, 8 \mathrm{~h}, 24 \mathrm{~h}$ and $48 \mathrm{~h}$ post infection, respectively.

datasets to deeply understand the biological process of macrophages and smooth or rough B. suis interaction. In the original study, the results have showed that macrophages presented different responses to smooth and rough $B$. suis and discovered some genes with larger change in gene expression level $(1,4)$. This partly explained the molecular mechanism of macrophages and B. suis interaction. However, the critical genes made subtle expression change would be lost. Therefore, in this study, we studied the critical pathways and their dynamical change in macrophage and B. suis interaction.

Toll-like receptor signaling pathway was found to be up-regulated during the 4 interaction stages. Toll-like re- ceptors (TLRs), the genes of specific families of pattern recognition receptors, are responsible for detecting microbial pathogens, generating innate immune responses and developing adaptive immunity, and have been extensively studies in macrophage as well as Brucella interaction (1820). These studies demonstrated that TLRs play an important role in host resistance to Brucella infection or cooperate with each other $(15,21)$. Such as TLR6, one member of TLRs is important for triggering an innate immune response against $B$. abortus and further cooperates with TRL2 to activate NF-kB signaling pathway (20). TLR4 and TLR9 also play a key role in macrophage and Brucella interaction (15). Such, we considered that the pathway related to im- 
A

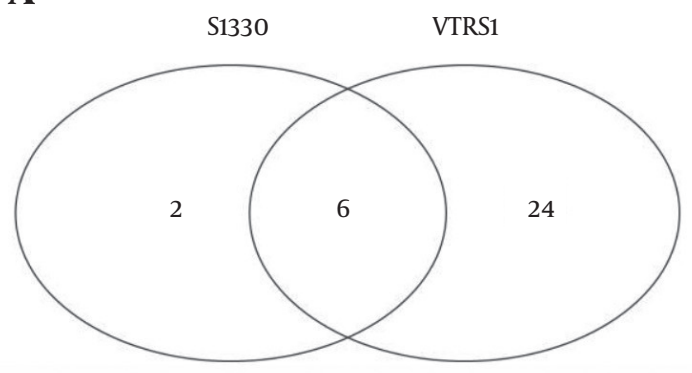

B

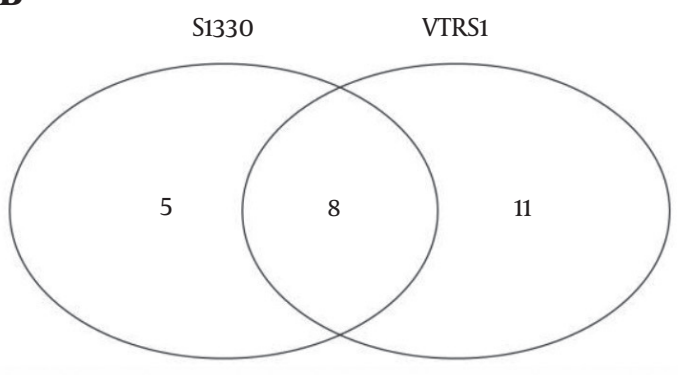

S1330 and VTRS1 are respectively represented common pathways identified post macrophages infected S1330 and VTRS1 B. suis. A, We separately detected 8 and 30 common pathways within macrophages infected S1330 and VTRS1 B. suis, and 6 common pathways were found; B, We separately detected 13 and 19 common pathways within macrophages infected S1330 and VTRS1 B. suis, and 8 common pathways were found.

mune system played a critical role in macrophage and $B$. suis interaction, and might serve as a potential target for novel antibiotic drug.

Beyond toll-like receptor signaling pathway upregulated, the other 2 up-regulated pathways related to immune system were RIG-I-like receptor signaling pathway and cytosolic DNA-sensing pathway. As similar to TLRs within toll-like receptor pathway, the receptors within the 2 pathways also belonged to specific families of pattern recognition receptors, which are respectively responsible for detecting viral pathogens as well as foreign DNA, and triggering innate immune responses. Currently, few published results directly showed RIG-I-like receptor signaling pathway, which was related to the interaction of macrophage and Brucella. Most published results proved that RIG-I-like receptor signaling pathway plays a vital role in RNA virus recognition (22-24). However, the functions of RIG-I-like receptor signaling pathway in defending against bacterial infection remain elusive. Lately, few studies showed that the role of RIG-I-like receptor signaling pathway in fighting against bacterial infection is a universal mechanism. RIG-I-like receptor signaling pathway is involved in the host immune response to several different types of bacterial pathogens, such as Yersinia pestis and so on (25). These studies provided theoretical supports for up-regulating RIG-I-like receptor signaling pathways in macrophage and B. suis interaction.

Cytosolic DNA-sensing pathway, a type of pathway generating innate immune response to DNA from DNA virus, bacteria or host cells, has been appreciated for many years where cytosolic DNA can evoke a Type I interferon response $(26,27)$. Some studies proved that DNA would be released into the cytoplasm from intracellular DNA-containing microbe such as Mycobacterium tuberculosis and Francisella tularensis during infections, and induced DNA immunity
$(28,29)$. Our results showed that cytosolic DNA-sensing pathway was up-regulated in the process of macrophage and $B$. suis interaction, which partly demonstrated that $B$. suis involved the similar interaction mechanisms as $M y$ cobacterium tuberculosis and Francisella tularensis during infections. That is to say, in macrophage and B. suis interaction, part B. suis killed may be disintegrated, and release DNA into macrophage to induce DNA immunity producing (30).

In up-regulated pathways, we also detected that rheumatoid arthritis pathway was up-regulated. Several studies have demonstrated that the brucellosis caused Brucella spp involved a diversity of clinical signs and symptoms including arthritis (31-35). Furthermore, some studies showed the relationship of brucellosis and rheumatic symptoms (32). However, these studies only described that some clinical symptoms of brucellosis patients were related to arthritis or rheumatoid arthritis. The mechanisms of arthritis caused Brucella are still unclear. In this study, we observed that many genes were significantly differently expressed within the rheumatoid arthritis pathway, in which many inflammatory and proinflammatory factors including interleukin 15, 6, 1beta, and tumor necrosis factor receptor and ligand superfamily members were up-regulated. Due to the fact that rheumatoid arthritis is caused by means of the body's immune system attacking the joints (36), rheumatoid arthritis pathway up-regulated may be one of the main reasons leading to joint pain. We speculated that the macrophages and $B$. suis interaction disturbed the pathway related to immune system, which makes the normal macrophage function disorder, and generates inflammatory and proinflammatory factors to promote the arthritis (37).

In down-regulated pathways, we found that the cell cycle pathway was down-regulated. A large number of stud- 
Table 3. Common Pathways Categories Identified by GSEA

\begin{tabular}{|c|c|c|c|c|}
\hline Entry & Pathway Name & Class & Gene Number $^{\mathrm{a}}$ & Genes $^{b}$ \\
\hline \multicolumn{5}{|l|}{ Up regulated } \\
\hline $04620^{c}$ & Toll-like receptor signaling pathway & Immune system & 14 & $\begin{array}{l}\text { Ikbke, Ccl5, Ccl3, Cd86, Ccl4, Tlr7, Pik3cg, } \\
\text { Fos, Pik3r1, Cd40, Tlr4, Il1b, Nfkbia, Tlr8 }\end{array}$ \\
\hline 04622 & RIG-I-like receptor signaling pathway & Immune system & 3 & Ikbke, Nfkbib, Nfkbia \\
\hline 04623 & Cytosolic DNA-sensing pathway & Immune system & 6 & Ikbke, Ccl5, Nfkbib, Ccl4, Illb, Nfkbia \\
\hline 05140 & Leishmaniasis & Infectious diseases & 6 & Fos, Ilib, Nfkbia, Nfkbib, Ptgs2, Tlr4 \\
\hline 05323 & Rheumatoid arthritis & Immune diseases & 6 & Cd86, Fos, Il1b, Ccl3, Ccl5, Tlr4 \\
\hline 04920 & Adipocytokine signaling pathway & Endocrine system & 5 & Socs3, Nfkbia, Nfkbib, Acsl3, Pck2 \\
\hline $\begin{array}{l}\text { Genes included in all } \\
\text { common up-regulated } \\
\text { pathways }\end{array}$ & & & 19 & $\begin{array}{l}\text { Ikbke, Ccl5, Ccl3, Cd86, Ccl4, Tlr7, Pik3cg, } \\
\text { Fos, Pik3r1, Cd40, Tlr4, Illb, Nfkbia, Tlr8, } \\
\text { Nfkbib, Ptgs2, Socs3, Acsl3, Pck2 }\end{array}$ \\
\hline \multicolumn{5}{|l|}{ Down regulated } \\
\hline 04110 & Cell cycle & Cell growth and death & 16 & $\begin{array}{l}\text { Atm, Ccnd1, Ccne2, Cdkn1a, Chek1, } \\
\text { Gadd45a, Anapc1, Gadd45b, Rbl1, Wee1, } \\
\text { Cdc6, Skp2, Gsk3b, Ccnh, E2f2, Atr }\end{array}$ \\
\hline 04114 & Oocyte meiosis & Cell growth and death & 4 & Ccne2, Itpr1, Anapc1, Prkacb \\
\hline 04914 & $\begin{array}{l}\text { Progesterone-mediated oocyte } \\
\text { maturation }\end{array}$ & Endocrine system & 4 & Anapc1, Pik3r1, Prkacb, Pik3cg \\
\hline 05210 & Colorectal cancer & Cancers & 7 & $\begin{array}{c}\text { Ccnd1, Fos, Msh6, Pik3r1, Tgfbr2, Pik3cg, } \\
\text { Gsk3b }\end{array}$ \\
\hline 05212 & Pancreatic cancer & Cancers & 5 & Ccnd1, Pik3r1, Tgfbr2, Pik3cg, E2f2 \\
\hline 05223 & Non-small cell lung cancer & Cancers & 6 & Ccnd1, Pik3r1, Sos1, Pik3cg, Stk4, E2f2 \\
\hline 03040 & Spliceosome & Transcription & 5 & $\begin{array}{c}\text { Srsfio, Srsf3, Prpfig, Prpf } 40 a, \text { U2surp, } \\
\text { Tra2a }\end{array}$ \\
\hline 04070 & $\begin{array}{l}\text { Phosphatidylinositol signaling } \\
\text { system }\end{array}$ & Signal transduction & 6 & $\begin{array}{c}\text { Itpr1, Pik3r1, Pikfyve, Pip4k2a, Pten, } \\
\text { Pik3cg }\end{array}$ \\
\hline $\begin{array}{l}\text { Genes included in all } \\
\text { common down-regulated } \\
\text { pathways }\end{array}$ & & & 34 & $\begin{array}{c}\text { Atm, Ccnd1, Ccne2, Cdkn1a, } \\
\text { Chek1,Gadd45a, Anapc1, Gadd45b, Rbl1, } \\
\text { Wee1, Cdc6, Skp2, Gsk3b, Ccnh, E2f2, Atr, } \\
\text { Itpr1, Prkacb, Pik3r1, Pik3cg, Fos, Msh6, } \\
\text { Tgfbr2, Sos1, Stk4, Srsfio, Srsf3, Prpfi1, } \\
\text { Prpf40a, U2surp, Tra2a, Pikfyve, } \\
\text { Pip4k2a, Pten }\end{array}$ \\
\hline
\end{tabular}

${ }^{a}$ Number of common genes included within the pathways.

${ }^{\mathrm{b}}$ Overlapping genes within the pathway.

'The number is represented pathway entry number, and the "mmu" is omitted in the front of the number.

ies showed that during the viral infection a number of viruses encode proteins to target cell cycle regulators and press cell cycle arrest (38-40). Furthermore, some studies showed that during bacterial infection the cell cycle of macrophage was arrested (41-43). For macrophage and Brucella interaction, some studies revealed that Brucella inhibited transcription of various host genes involved in cell cycling (16), which indicated that cell cycle pathway within macrophage might be down-regulated in macrophage and Brucella interaction. Our GSEA results showed that cell cycle pathway would be down-regulated in B. suis and macrophage interaction. Down-regulated cell cycle would subvert the cellular machinery that controlled replication of macrophage (39), which would benefit bacterial survival and growth within the host (44).

Beyond common critical pathways discussed above, we observed that metabolic pathways were down-regulated in the majority of interaction stages. The metabolic pathways belong to a global pathway involved in a serious of chemical reactions of material metabolism (45). When infection, some studies showed that pathways or members related to lipid, sugar, and protein metabolism were changed (4648). It is easy to understand that pathogen infection will result in metabolic disturbance of host or cell $(49,50)$.

The interaction of macrophage and B. suis is extremely intricate. The infection process involves a series of im- 
A

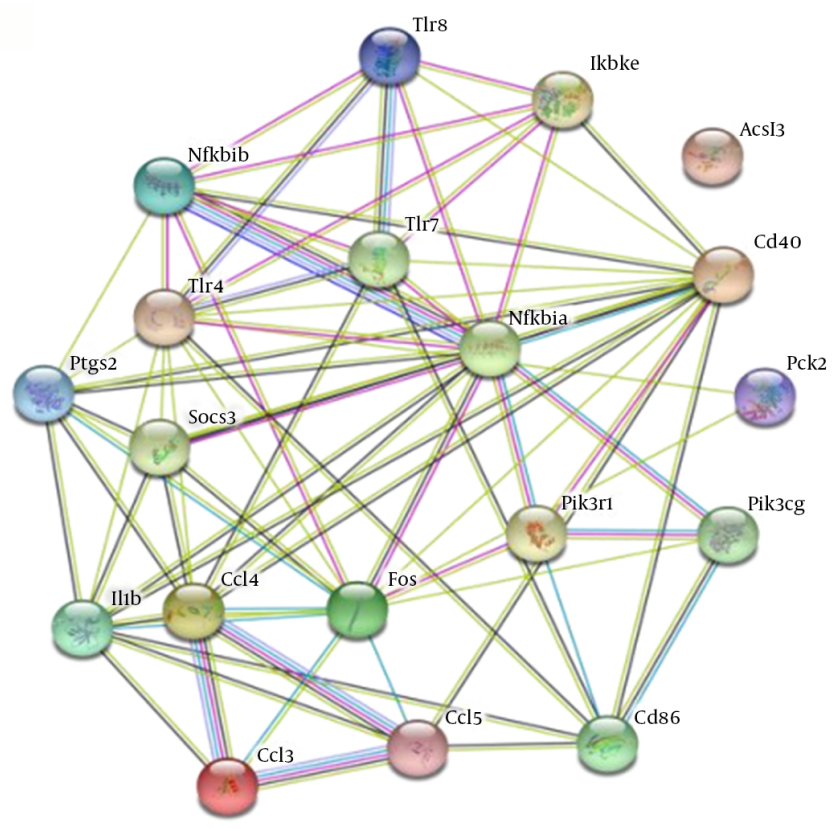

B

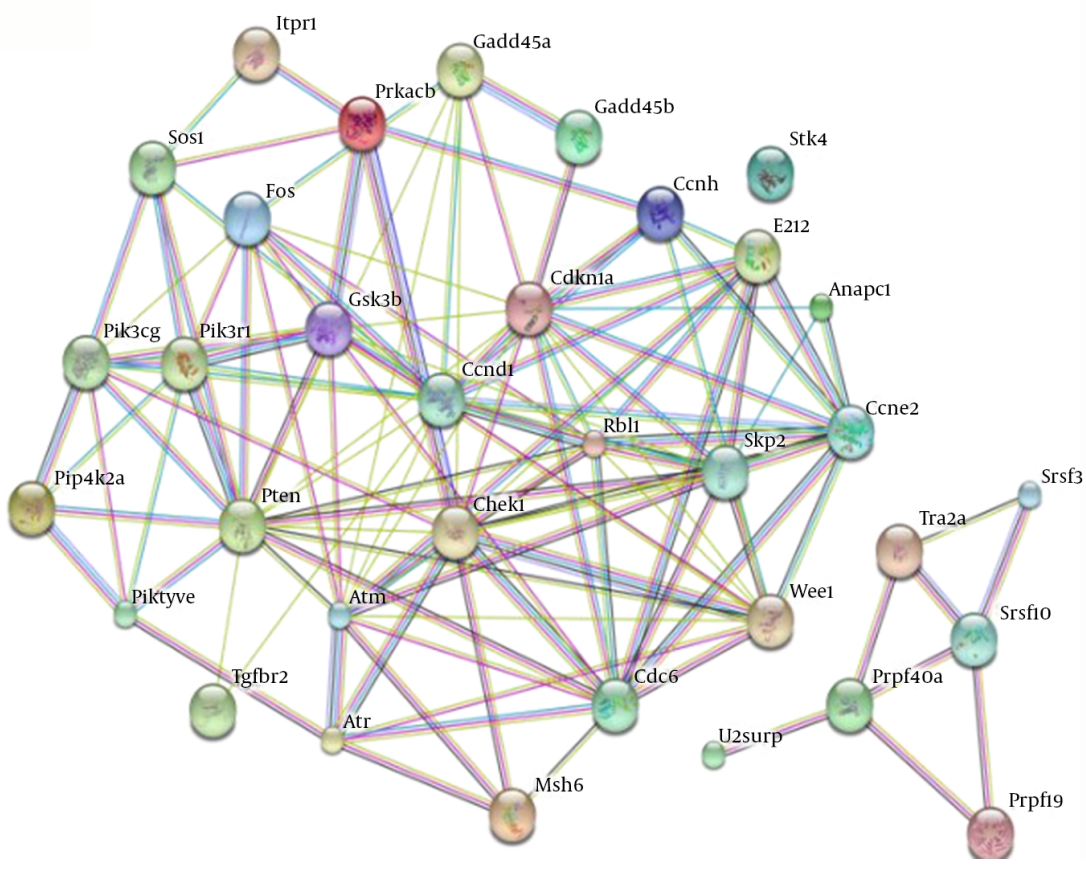

A, Association network of gene sumset in common up-regulated pathways; B, Association network of gene sumset in common down-regulated pathways.

munological and inflammatory reaction events within macrophage against B. suis infection. Inversely, B. suis have evolved a unique strategy to modify and create a novel intracellular environment for surviving and multiplying. A deeper understanding of the interaction mechanisms can be reached by focusing on regulation of gene sets rather than on a large number of single genes. By means of standardized microarray preprocessing and GSEA, we found 
Figure 5. Dynamical Change of Common Critical Pathways

\begin{tabular}{lllllll}
\hline & \multicolumn{8}{c}{ Time Point Post Infection } \\
\cline { 2 - 6 } Pathway Name & & oh & $4 h$ & $8 h$ & $24 h$ & $48 h$ \\
\hline
\end{tabular}

\section{S1330 Strain*}

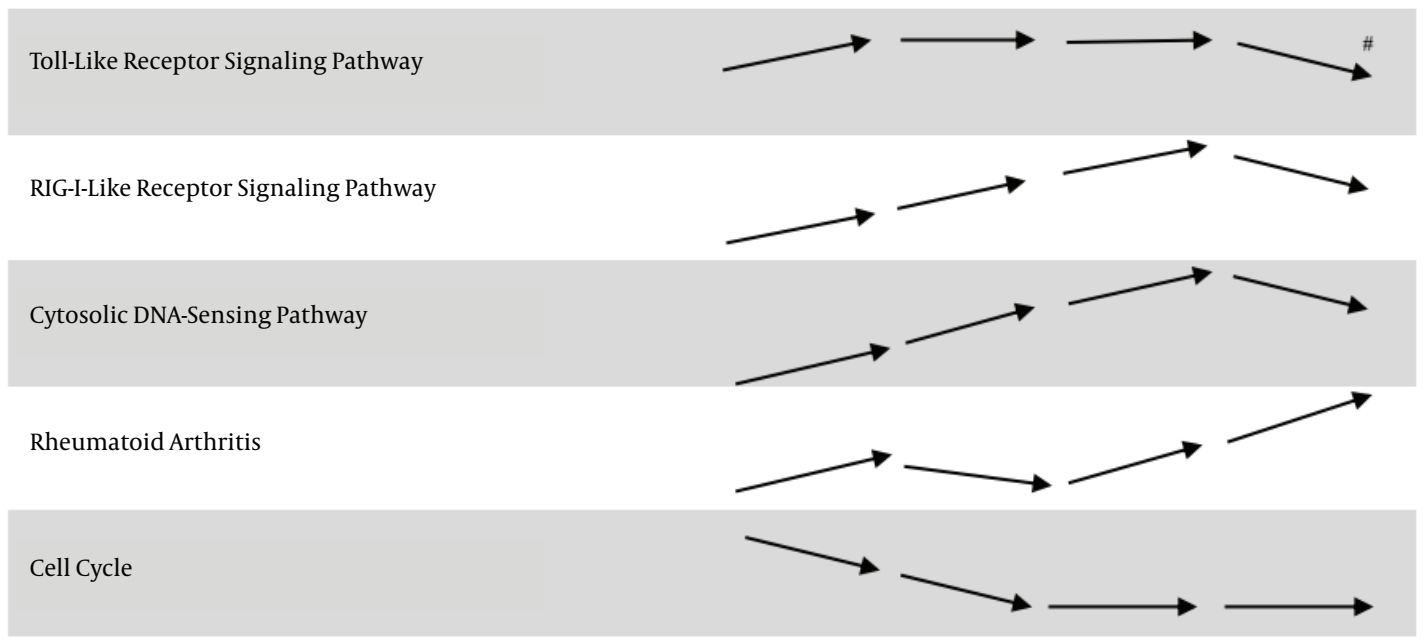

VTRS1 Strain

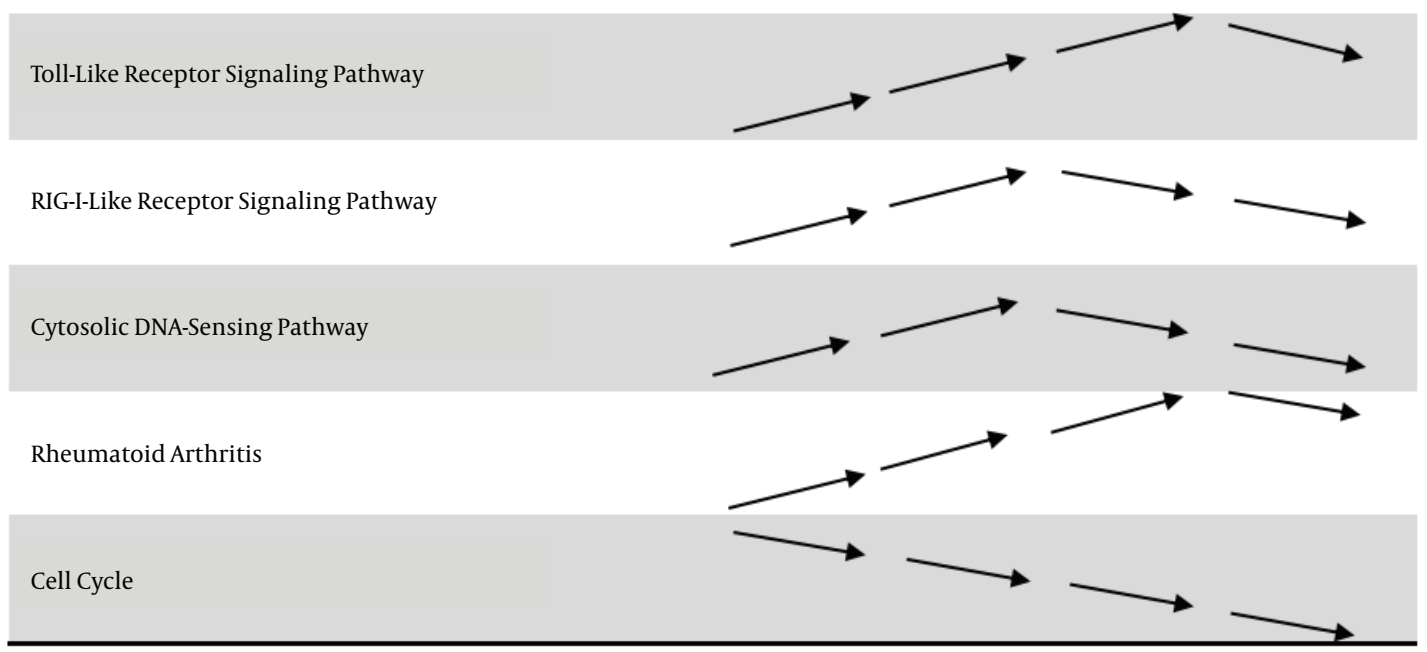

*S1330 strain and VTRS1 strain are represented murine macrophage-like J774.A1 cells infected by smooth virulent B. suis strain and rough attenuated B. suis strain, respectively. \# Arrow line is represented the regulated change of over-represented pathway. The parallel arrow shows that the pathway has no significant change in the latter infection stage than that in the former stage. The arrow line with raising end shows that the pathway is significantly up-regulated in the latter infection stage than that in the former stage. The arrow line with dropping end shows that the pathway is significantly down-regulated in the latter infection stage than that in the former stage.

the concordance to identify many biological mechanisms involved in interaction of macrophage and B. suis in some infection stages. The identified corresponding pathways will provide some important insights into immunologi- 
cal reactions within macrophage post infected B. suis. Further, these results will provide some clues for discovering novel wide-spectrum antibiotic drug targets for helping to more efficiently prevent and control brucellosis. Next, more studies will focus on the specific genes within related pathways and gene interaction to improve the understanding of macrophage and B. suis interaction.

\section{Conclusions}

Through pathway analysis based GSEA, we found some critical biological pathways involved in the process of macrophage and B.suis interaction. These results will contribute to deeply understand the interaction mechanisms, and help to efficiently prevent and control brucellosis. Nevertheless, the number of the array datasets used only contains 2 datasets and the array datasets have a limited set of probes of the mouse genome. Thus, further studies with arrays covering the entire repertoire of mouse genes will be needed to update the current results.

\section{Acknowledgments}

The authors are grateful to Kunming University of Science and Technology, Yunnan Provincial Science and Technology Department, as well as National Natural Science Foundation of China for their financial support of this work.

\section{Footnotes}

Authors' Contribution: Jing Hu searched the microarray from NCBI GEO database. Qiang Chen and Hongbo Zhao performed the data processing and GSEA with the help of Tonglian Wang, Yuzhu Song, Qinqin Han, Jinyang Zhang. Qiang Chen wrote the manuscript. Tao Shou, Fan Zhang and Xueshan Xia revised the manuscript.

Financial Disclosure: The authors declare no personal or professional conflicts of interest with any aspect of this study.

Funding/Support: This work was supported by the Fund for Fostering Talents of Kunming University of Science and Technology (No. KKSY201526053), Yunnan province Applied Basic Research Projects (No. 2016FB146) and National Natural Science Foundation of China (No. 31660655).

\section{References}

1. Chen F, Ding X, Ding Y, Xiang Z, Li X, Ghosh D, et al. Proinflammatory caspase-2-mediated macrophage cell death induced by a rough attenuated Brucella suis strain. Infect Immun. 2011;79(6):2460-9. doi: 10.1128/IAI.00050-11. [PubMed: 21464087].
2. Whatmore AM. Current understanding of the genetic diversity of Brucella, an expanding genus of zoonotic pathogens. Infect Genet Evol. 2009;9(6):1168-84. doi: 10.1016/j.meegid.2009.07.001. [PubMed: 19628055].

3. Liautard JP, Gross A, Dornand J, Kohler S. Interactions between professional phagocytes and Brucella spp. Microbiologia. 1996;12(2):197-206. [PubMed: 8767704].

4. Chen F, He Y. Caspase-2 mediated apoptotic and necrotic murine macrophage cell death induced by rough Brucella abortus. PLoS One. 2009;4(8):e6830. doi: 10.1371/journal.pone.0006830. [PubMed: 19714247].

5. He Y, Reichow S, Ramamoorthy S, Ding X, Lathigra R, Craig JC, et al. Brucella melitensis triggers time-dependent modulation of apoptosis and down-regulation of mitochondrion-associated gene expression in mouse macrophages. Infect Immun. 2006;74(9):5035-46. doi: 10.1128/IAI.01998-05. [PubMed: 16926395].

6. Pei J, Kahl-McDonagh M, Ficht TA. Brucella dissociation is essential for macrophage egress and bacterial dissemination. Front Cell Infect Microbiol. 2014;4:23. doi: 10.3389/fcimb.2014.00023. [PubMed: 24634889].

7. Faherty CS, Maurelli AT. Staying alive: bacterial inhibition of apoptosis during infection. Trends Microbiol. 2008;16(4):173-80. doi: 10.1016/j.tim.2008.02.001. [PubMed:18353648].

8. Gao LY, Kwaik YA. The mechanism of killing and exiting the protozoan host Acanthamoeba polyphaga by Legionella pneumophila. Environ Microbiol. 2000;2(1):79-90. [PubMed: 11243265].

9. Mootha VK, Lindgren CM, Eriksson KF, Subramanian A, Sihag S, Lehar J, et al. PGC-1alpha-responsive genes involved in oxidative phosphorylation are coordinately downregulated in human diabetes. Nat Genet. 2003;34(3):267-73. doi: 10.1038/ng1180. [PubMed: 12808457].

10. Subramanian A, Tamayo P, Mootha VK, Mukherjee S, Ebert BL, Gillette MA, et al. Gene set enrichment analysis: A knowledge-based approach for interpreting genome-wide expression profiles. Proc Nation Acad Sci. 2005;102(43):15545-50. doi:10.1073/pnas.0506580102.

11. Gentleman RC, Carey VJ, Bates DM, Bolstad B, Dettling M, Dudoit S, et al. Bioconductor: open software development for computational biology and bioinformatics. Genome Biol. 2004;5(10):R80. doi: 10.1186/gb-2004-5-10-r80.

12. Irizarry RA. Exploration, normalization, and summaries of high density oligonucleotide array probe level data. Biostatistics. 2003;4(2):249-64. doi: 10.1093/biostatistics/4.2.249.

13. Gautier L, Cope L, Bolstad BM, Irizarry RA. affy-analysis of Affymetrix GeneChip data at the probe level. Bioinformatics. 2004;20(3):307-15. doi: 10.1093/bioinformatics/btg405.

14. Zhao H, Huang M, Chen Q, Wang Q, Pan Y. Comparative gene expression analysis in mouse models for identifying critical pathways in mammary gland development. Breast Cancer Res Treat. 2011;132(3):969-77. doi: 10.1007/s10549-011-1650-8.

15. Gao G, Xu J. Important Biology Events and Pathways in Brucella Infection and Implications for Novel Antibiotic Drug Targets. Crit Rev Eukaryotic Gene Express. 2013;23(1):65-76. doi: 10.1615/CritRevEukarGeneExpr.2013006580.

16. Eskra L, Mathison A, Splitter G. Microarray Analysis of mRNA Levels from RAW264.7 Macrophages Infected with Brucella abortus. Infect Immun. 2003;71(3):1125-33. doi:10.1128/iai.71.3.1125-1133.2003.

17. Cha SB, Lee WJ, Shin MK, Jung MH, Shin SW, Yoo AN, et al. Early transcriptional responses of internalization defective Brucella abortus mutants in professional phagocytes, RAW 264.7. BMC Genomics. 2013;14(1):426. doi:10.1186/1471-2164-14-426.

18. Campos MA, Rosinha GMS, Almeida IC, Salgueiro XS, Jarvis BW, Splitter GA, et al. Role of Toll-Like Receptor 4 in Induction of Cell-Mediated Immunity and Resistance to Brucella abortus Infection in Mice. Infect Immun. 2003;72(1):176-86. doi: 10.1128/iai.72.1.176-186.2004. 
19. Pei J, Ding X, Fan Y, Rice-Ficht A, Ficht TA. Toll-like receptors are critical for clearance of Brucella and play different roles in development of adaptive immunity following aerosol challenge in mice. Front Cell Infect Microbiol. 2012;2 doi:10.3389/fcimb.2012.00115.

20. de Almeida LA, Macedo GC, Marinho FAV, Gomes MTR, Corsetti PP, Silva AM, et al. Toll-Like Receptor 6 Plays an Important Role in Host Innate Resistance to Brucella abortus Infection in Mice. Infect Immun. 2013;81(5):1654-62. doi:10.1128/iai.01356-12.

21. Ojcius D, Barquero-Calvo E, Chaves-Olarte E, Weiss DS, Guzmán-Verri C, Chacón-Díaz C, et al. Brucella abortus Uses a Stealthy Strategy to Avoid Activation of the Innate Immune System during the Onset of Infection. PLoS ONE. 2007;2(7):e631. doi: 10.1371/journal.pone.0000631.

22. Kell AM, Gale M. RIG-I in RNA virus recognition. Virology. 2015;479480:110-21. doi: 10.1016/j.virol.2015.02.017.

23. Sato S, Li K, Kameyama T, Hayashi T, Ishida Y, Murakami S, et al. The RNA Sensor RIG-I Dually Functions as an Innate Sensor and Direct Antiviral Factor for Hepatitis B Virus. Immunity. 2015;42(1):123-32. doi: 10.1016/j.immuni.2014.12.016.

24. Fan X, Dong S, Li Y, Ding S, Wang M. RIG-I-dependent antiviral immunity is effective against an RNA virus encoding a potent suppressor of RNAi. Biochem Biophys Res Commun. 2015;460(4):1035-40. doi: 10.1016/j.bbrc.2015.03.145.

25. Du Z, Yang H, Tan Y, Tian G, Zhang Q, Cui Y, et al. Transcriptomic response to Yersinia pestis: RIG-I like receptor signaling response is detrimental to the host against plague. J Genet Genomics. 2014;41(7):379-96. doi:10.1016/j.jgg.2014.05.006. [PubMed: 25064677].

26. Dempsey A, Bowie AG. Innate immune recognition of DNA: A recent history. Virology. 2015;479-480:146-52. doi:10.1016/j.virol.2015.03.013.

27. Keating SE, Baran M, Bowie AG. Cytosolic DNA sensors regulating type I interferon induction. Trends Immunol. 2011;32(12):574-81. doi: 10.1016/j.it.2011.08.004.

28. Manzanillo P S, Shiloh M U, Portnoy D A, Cox JS. Mycobacterium Tuberculosis Activates the DNA-Dependent Cytosolic Surveillance Pathway within Macrophages. Cell Host Microbe. 2012;11(5):469-80. doi: 10.1016/j.chom.2012.03.007.

29. Fernandes-Alnemri T, Yu JW, Juliana C, Solorzano L, Kang S, Wu J, et al. The AIM2 inflammasome is critical for innate immunity to Francisella tularensis. Nat Immunol. 2010;11(5):385-93. doi:10.1038/ni.1859.

30. Celli J. Intracellular localization of Brucella abortus and Francisella tularensis in primary murine macrophages. Bacterial Pathogenesis Methods Protocols. 2008:133-45.

31. Khateeb MI, Araj GF, Majeed SA, Lulu AR. Brucella arthritis: a study of 96 cases in Kuwait. Ann Rheumatic Dis. 1990;49(12):994-8. doi: 10.1136/ard.49.12.994.

32. Norton WL. Brucellosis and rheumatic syndromes in Saudi Arabia. Ann Rheumatic Dis. 1984;43(6):810-5. doi:10.1136/ard.43.6.810.

33. Porat S, Shapiro M. Brucella arthritis of the sacro-iliac joint. Infection. 1984;12(3):205-7. doi: 10.1007/bf01640901.

34. Kooraki S, Mohazab RA, Zamani N, Matloob R, Hayatbakhsh M, Raeeskarami SR, et al. Epidemiological and clinical features of Brucella arthritis in 24 children. Ann Saudi Med. 2011;31(3):270. doi:
10.4103/0256-4947.81543.

35. Wong TM, Lou N, Jin W, Leung F, To M, Leung F. Septic arthritis caused by Brucella melitensis in urban Shenzhen, China: a case report. J Med Case Rep. 2014;8(1) doi: 10.1186/1752-1947-8-367.

36. McInnes IB, Schett G. The Pathogenesis of Rheumatoid Arthritis. N England J Med. 2011;365(23):2205-19. doi:10.1056/NEJMra1004965.

37. Smolen JS, Redlich K, Zwerina J, Aletaha D, Steiner G, Schett G. ProInflammatory Cytokines in Rheumatoid Arthritis: Pathogenetic and Therapeutic Aspects. Clin Rev Allergy Immunol. 2005;28(3):239-48. doi: 10.1385/criai:28:3:239.

38. Chen AY, Qiu J. Parvovirus infection-induced cell death and cell cycle arrest. Future Virol. 2010;5(6):731-43. doi: 10.2217/fvl.10.56.

39. Bagga S, Bouchard MJ. Cell Cycle Regulation During Viral Infection. Methods Mol Biol. 2014;1170:165-227.doi:10.1007/978-1-4939-0888-2_10.

40. Kannan RP, Hensley LL, Evers LE, Lemon SM, McGivern DR. Hepatitis C Virus Infection Causes Cell Cycle Arrest at the Level of Initiation of Mitosis. JVirol. 2011;85(16):7989-8001. doi: 10.1128/jvi.00280-11.

41. Kasai H, Nakashima K, Yokota M, Nishihara T. The G1 cell cycle arrest of macrophages infected withAggregatibacter actinomycetemcomitans. Oral Dis. 2010;16(3):305-9. doi: 10.1111/j.1601-0825.2010.01667.x.

42. Coelho C, Tesfa L, Zhang J, Rivera J, Goncalves T, Casadevall A. Analysis of Cell Cycle and Replication of Mouse Macrophages after In Vivo and In Vitro Cryptococcus neoformans Infection Using Laser Scanning Cytometry. Infect Immu. 2012;80(4):1467-78. doi:10.1128/iai.06332-11.

43. Tran G, Nhieu V, Arbibe L. Genetic reprogramming of host cells by bacterial pathogens. F1000 Biol Rep. 2009;1 doi: 10.3410/b1-80.

44. Kim M, Ashida H, Ogawa M, Yoshikawa Y, Mimuro H, Sasakawa C. Bacterial Interactions with the Host Epithelium. Cell Host Microbe. 2010;8(1):20-35. doi:10.1016/j.chom.2010.06.006.

45. Kanehisa M, Goto S. KEGG: kyoto encyclopedia of genes and genomes. Nucleic Acids Res. 2000;28(1):27-30. [PubMed:10592173].

46. Sanchez V, Dong JJ. Alteration of lipid metabolism in cells infected with human cytomegalovirus. Virology. 2010;404(1):71-7. doi: 10.1016/j.virol.2010.04.026. [PubMed: 20552728].

47. Wen C, He X, Ma H, Hou N, Wei C, Song T, et al. Hepatitis C virus infection downregulates the ligands of the activating receptor NKG2D. Cell Mol Immunol. 2008;5(6):475-8. doi: 10.1038/cmi.2008.60. [PubMed: 19118515].

48. Li H, Zhu W, Zhang L, Lei H, Wu X, Guo L, et al. The metabolic responses to hepatitis $B$ virus infection shed new light on pathogenesis and targets for treatment. Sci Rep. 2015;5:8421. doi: 10.1038/srep08421. [PubMed: 25672227].

49. Howard AA, Floris-Moore M, Arnsten JH, Santoro N, Fleischer N, Lo Y, et al. Disorders of glucose metabolism among HIV-infected women. Clin Infect Dis. 2005;40(10):1492-9. doi: 10.1086/429824. [PubMed: 15844072].

50. Larson R, Capili B, Eckert-Norton M, Colagreco JP, Anastasi JK. Disorders of glucose metabolism in the context of human immunodeficiency virus infection. J Am Acad Nurse Pract. 2006;18(3):92-103. doi: 10.1111/j.1745-7599.2006.00109.x. [PubMed:16499742]. 